\title{
Prolonged Low-Level Exposure to the Aroma of Peppermint Essential Oil Enhances Aspects of Cognition and Mood in Healthy Adults
}

\author{
Lauren Hoult, Laura Longstaff, Mark Moss* \\ Department of Psychology, Faculty of Health and Life Sciences, Northumbria University, Newcastle upon Tyne, UK \\ Email: *mark.moss@northumbria.ac.uk
}

How to cite this paper: Hoult, L., Longstaff, L. and Moss, M. (2019) Prolonged Low-Level Exposure to the Aroma of Peppermint Essential Oil Enhances Aspects of Cognition and Mood in Healthy Adults. American Journal of Plant Sciences, 10, 1002-1012.

https://doi.org/10.4236/ajps.2019.106072

Received: March 1, 2019

Accepted: June 18, 2019

Published: June 21, 2019

Copyright $\odot 2019$ by author(s) and Scientific Research Publishing Inc. This work is licensed under the Creative Commons Attribution International License (CC BY 4.0).

http://creativecommons.org/licenses/by/4.0/

\section{c) (i) Open Access}

\begin{abstract}
The possible positive impact of aromas of plant essential oils is an area with a long history and one that is receiving increasing interest from modern science. Previous research has demonstrated beneficial effects of acute exposure on a range of variables. The current study set out to investigate for the first time whether extended exposure to the aroma of peppermint essential oil could provide benefits to healthy young adults. One hundred participants were randomly assigned to either wear a peppermint infused non-transdermal skin patch or a blank patch, for a period of six hours during which time they went about their normal daily routine. Cognitive and mood assessments were completed and a multivariate analysis of variance indicated a significant difference to exist between the two groups when all variables were analysed together. Further analyses on the individual variables revealed small to medium positive effects of peppermint aroma on aspects of memory and attention and feelings of subjective alertness. These findings add to a growing body of evidence that suggests aroma of peppermint essential oil may act as a positive and easy intervention delivering beneficial objective and subjective outcomes. The findings are discussed in terms of putative mechanisms that might underpin the effects with a focus on possible pharmacological influences.
\end{abstract}

\section{Keywords}

Aromas, Plant, Essential Oils

\section{Introduction}

Peppermint (Mentha piperita Lamiaceae) is a native European perennial herb now cultivated worldwide for use as a food flavouring and aromatic additive in 
perfume and household products. As is the case for many herbs, Peppermint has a well-documented history of use as a therapeutic and life enhancing plant. Furthermore, many of these applications are still recognised today, and the some of the pharmacology that underpins them has been identified; see McKay and Blumberg [1] for a review. The administration of peppermint is not limited to oral preparations however. The extraction of plant essential oils provides the opportunity to deliver aromas as possible therapeutics, following the age old tradition of aromatherapy [2]. Studies have demonstrated the positive impact of peppermint aroma on postoperative nausea following c-section [3], stress [4], and nasal congestion [5].

In recent years, a small body of research has also delivered scientific investigations into the effects of peppermint aroma on a number of non-therapeutic variables, such as its limiting effects on daytime sleepiness [6]. Mahachandra and Garnaby [7] suggest that the stimulating properties of peppermint aroma might be utilised in in-car fragrance dispersal units to reduce driver sleepiness and have a positive impact on associated vehicle accidents. This proposal is supported by evidence that peppermint aroma improved performance in sleep deprived drivers to unexpected situations in a simulation experiment [8]. In the field of sport and exercise the inhalation of peppermint aroma has been demonstrated to increase in running speed, hand grip strength, and number of push-ups, but not to impact upon on skill related tasks [9]. However, a later study found no effect of peppermint aroma on the physiological parameters: oxygen consumption, minute ventilation or respiratory exchange ratio during acute exercise [10]. Basevitch et al. [11] published data suggesting that any impact on performance measures may be a consequence of the aromas effect on psychological rather than physiological variables. This contention is further supported by a study that reported decreased anxiety levels and improved performance (accuracy and consistency) on a dart throwing task for participants in a peppermint aroma condition [12].

Considering psychological effects further, McCombs, Raudenbush, Bova, and Sappington [13] found that peppermint aroma delivered an experience of less mental demand, perceived effort, and anxiety during video game play that also displayed better performance compared to a no aroma control condition. A study into economic problem solving and decision making demonstrated that peppermint aroma improved participants overall mood during the task and the authors argue this delivered better results on the economic task [14]. In terms of underlying cognitive operations Moss, Hewitt, Moss, and Wesnes [15] investigated the acute impact of exposure to peppermint aroma compared to that of ylang-ylang and a no aroma control. Peppermint aroma was found to positively impact on accuracy of long-term memory compared to both ylang-ylang and no aroma conditions. A number of other differences were found between the two aroma conditions but not between peppermint and no aroma. In terms of mood, peppermint also produced a small increase in alertness, although this was not 
significantly different to the control group and as such the change in cognition is discounted as being a consequence of increased subjective arousal and associated engagement with the tasks. Recent research has suggested that peppermint essential oil with high levels of menthol/menthone displays acetylcholinesterase inhibitory and nicotinic receptor binding properties, and delivers enhancement in cognitive performance when administered orally [16]. It is possible that similar mechanisms may underpin aroma based effects of peppermint, as previously suggested for sage [17] and rosemary [18].

There is an increasingly growing market for aroma-based products designed for day-long or regular prolonged use, but evidence of beneficial effects is currently sparse. Morris [19] reported reductions in anger-frustration and negative responses about the future in healthy participants following a 14-day lavender bath intervention compared to controls, and suggest further investigation is warranted. More recently, an over-the-counter aromatherapy device was found to reduce postoperative nausea over a 24-hour period [20]. The current study aimed to extend our knowledge of the potential impact of extended exposure to low levels of peppermint essential oil aroma on mood and cognition in healthy adults.

\section{Method}

\subsection{Design}

A single factor independent groups design was employed. The independent variable was the presence or absence of peppermint essential oil aroma applied to a patch worn by the participants. The dependent variables were cognitive performance measures: word recall, prospective memory, verbal fluency and letter cancellation, and subjective mood.

\subsection{Participants}

One hundred participants were recruited via poster adverts and each was paid $£ 10$ each for taking part in the study. All [participants completed a standard health screen questionnaire and none were excluded on this basis. The peppermint aroma group consisted of 23 males (mean age $=25.09$ years, $S D=8.53$ ) and 27 females (mean age $=27.37$ years, $S D=11.04$ ). The no aroma control group consisted of 27 males (mean age $=28.00$ years, $\mathrm{SD}=11.08$ ) and 23 females (mean age $=26.00$ years, $\mathrm{SD}=9.57$ )

\subsection{Materials}

\subsubsection{Aroma Patches}

Blank and peppermint infused aroma patches were sourced from Bioesse Technologies LLC, Minnetonka, USA and applied in accordance with the manufacturer's instructions.

\subsubsection{Cognitive Tasks}

Word Recall-Fifteen words were presented one at a time on a computer 
screen. Each word had a screen duration of one second and each word was separated by a two-second blank screen. Immediate word recall was assessed immediately after the presentation. Delayed word recall was assessed when participants returned to the lab for the second visit.

Prospective Memory-Time based prospective memory was assessed by giving participants a time at which to text the researcher to confirm that they were returning for the second (afternoon) session. The length of time in minutes that the participant deviated from the agreed time provided a measure of error in this variable. Event based prospective memory was assessed by giving participants an envelope in the morning session and asking them to give it back to the researcher just before completing the mood scales in the afternoon session. Participants who returned the envelope spontaneously at the appropriate stage of the procedure received a score of 2 , those who handed it over at the wrong point or handed it over following a prompt received a score of 1 . Any participant who failed to bring the envelope with them or didn't return it until asked to do so at the very end of the session received zero.

Letter Cancellation-A sheet of A4 paper with 26 printed rows of lower case letters was produced. Each row contained 15 letters. A total of 34 letter " $e$ " were present amongst the stimuli letters and these constituted the target letter. Participants were required to scan the sheet and strike though with a pencil as many instances of the target letter as they could in 30 seconds. The total number of correct targets detected being the dependent variable. Piloting of the task ensured that no one would complete the task fully before the time limit elapsed. Letter cancellation tasks are widely used as a simple measure of attention (Uttl \& Pilkenton-Taylor 2001).

Verbal Fluency-Participants were required to write down as many words as they could beginning with the letter "S". A time limit of one-minute was placed on the test. Verbal fluency is considered to be a reliable assessment of executive functioning [21].

\subsubsection{Mood Measures}

Visual analogue mood scales (Bond \& Lader 1974) were completed at the start of the morning session and at the end of the afternoon session. The scales are made up of 16 pairs of bipolar words, with each pair separated by a $100 \mathrm{~mm}$ line. Participants are asked to mark each line to indicate how they feel at that point in time on each dimension as defined by the two words. The measures are then combined to produce scores on the three mood dimensions Alert, Content and Calm. To assess the change in mood over time the morning session scores were subtracted from the afternoon session scores such that a positive value indicated an increase in that measure across the course of the study day.

\subsection{Procedure}

Participants were required to attend the lab at two points on the same day. The first session took place between 9:00 and 10:00am, with individual participant 
appointments determined by mutual convenience. The second session was determined as six hours after the morning session. On arrival at the lab participants were fully informed about the aims of the study, and consent obtained. The mood scales were then completed, and the aroma patch applied. Participants were not told which condition they were in, although those in the peppermint condition would have been aware of the aroma. Participants were then given the instructions for the prospective memory tasks to read and any questions answered. Five minutes after the application of the patch, the immediate word recall task was completed. Participants were then free to leave the lab and continue their normal daily activities before returning to the lab in the afternoon between 3:00 and 4:00pm. On returning to the lab, participants completed the delayed word recall, letter cancellation, and verbal fluency tasks, and performance on the prospective memory tasks recorded. Finally, the mood scales were completed prior to the aroma patches being removed and participants debriefed.

The study gained full ethical approval from the Department of Psychology ethics committee at Northumbria University, UK, prior to the start of testing.

\section{Results}

Due to the large number of dependent variables, data were analysed initially via a one way multivariate analysis of variance (Manova) in order to identify if a global difference existed between the conditions. The analysis revealed a significant difference to exist between the two conditions for the combined dependent variables Wilks' Lambda $=0.832, \mathrm{~F}(9,90)=2.025, \mathrm{p}=0.045$, partial Eta squared $=0.168$.

Consideration of the individual dependent variables is made on the basis of effect size conventions as established by Cohen [22] rather than statistical significance as the large number of dependent variables means Bonferroni correction will lead anything with a $\mathrm{p}$ value of greater than .005 to be deemed non-significant. Descriptive statistics for all dependent variables are presented in Table 1.

\section{Discussion}

The results of this study demonstrate the potential for extended low-level exposure to the aroma of peppermint essential oil to impact on cognition and mood in healthy young adults. Small to medium effect sizes (based on Cohen's criteria) in favour of peppermint aroma were found for immediate and delayed word recall, event-based prospective memory and letter cancellation. In addition, a medium sized effect was found for the increase in the subjective mood measure of alertness.

With regard to the impact on memory performance, previous research [15] identified smaller benefits from acute exposure to peppermint on immediate word recall $(d=0.23)$ and delayed word recall $(d=0.26)$ than those observed here. A small positive effect $(\mathrm{d}=0.20)$ has also been reported for a non-verbal 
Table 1. Mean (standard deviation) values for all dependent variables. IWR $=$ immediate word recall number correct; $\mathrm{EPM}=$ event based prospective memory score; $\mathrm{TPM}=$ time based prospective memory, deviation from scheduled time in minutes; DWR = delayed word recall number correct; VF = verbal fluency, number of words generated; LC = letter cancellation, number of targets detected. Values for Alert, Content and Calm represent changes from the morning to afternoon assessments.

\begin{tabular}{ccccc}
\hline & Peppermint & Control & Uncorrected $\mathrm{p}$ & Cohen's d \\
\hline IWR & $8.94(2.68)$ & $7.88(2.15)$ & 0.032 & 0.44 \\
EPM & $1.00(0.88)$ & $0.66(0.82)$ & 0.049 & 0.40 \\
TPM & $6.62(3.28)$ & $7.68(3.97)$ & 0.149 & 0.29 \\
DWR & $5.82(2.41)$ & $4.962 .08)$ & 0.059 & 0.38 \\
VF & $14.08(4.65)$ & $13.50(4.16)$ & 0.513 & 0.13 \\
LC & $23.48(6.40)$ & $20.62(5.17)$ & 0.016 & 0.49 \\
Alert & $4.08(21.71)$ & $-6.96(19.25)$ & 0.008 & 0.54 \\
Content & $-3.13(22.31)$ & $0.71(20.66)$ & 0.374 & 0.18 \\
Calm & $-4.37(20.44)$ & $-7.78(15.64)$ & 0.351 & 0.19 \\
\hline
\end{tabular}

memory task under conditions of acute peppermint aroma exposure [23]. The findings of the current study suggest therefore that duration of exposure may be an important factor in terms of beneficial outcomes in long term memory. Considering prospective memory, small beneficial effects of acute peppermint aroma exposure have been found for both "time based" and "event based" measures assessed using the Camprompt test [24]. The results reported here are consistent with these and suggest no added benefit of extended exposure. However, it is worth noting that the prospective memory tasks employed here-although fewer in number-do perhaps possess greater ecological validity than the lab-based Camprompt assessment, and require participants to do something based on an "event" or "time" some hours later when they are not in the lab. It may be that the prospective memory tasks used here are more difficult than the lab based ones, and as such do not generate differences in effect in response to extended exposure of the size observed for the long term memory tasks.

The impact on the letter cancellation task reported here suggests improved attentional processing following extended exposure to peppermint. The effect size (d) of 0.49 is considerably larger than the 0.18 found for the speed of attention factor previously under conditions of acute exposure [15]. Although the different nature of the tasks may contribute to this difference to some extent, the potential differential impact of extended versus acute exposure must again be considered seriously. Furthermore, the recorded increase in the subjective measure of alertness here coincides with the improved performance on the attention based letter cancellation task. The positive impact of peppermint aroma on measures of attention have also been reported elsewhere. Barker et al. [23] reported a large effect size $(d=0.8)$ on a clerical task requiring high levels of at- 
tention when completed under peppermint aroma exposure compared to a no aroma control. The authors suggest this is a consequence of increased physiological arousal producing increased awareness and attention to detail, although no measures of physiology or subjective mood were recorded. Norrish and Dwyer [6] investigated the effects of peppermint aroma on an objective measure of daytime sleepiness-the Pupillary Unrest Index-and found a very large effect $(\mathrm{d}=1.6)$ in favour of peppermint aroma when compared to no aroma over an 11-minute recording period. This has been further reinforced by the measurement of subjective state during driving simulations where peppermint produced increases in alertness and reductions in perceptions of demand and fatigue [25]. However, although the authors state that measures of cognitive performance were assessed, only a subjective evaluation of performance is presented. The lack of any observable effect for this measure is difficult to meaningfully compare with objective measures of the kind reported for the current study. McCombs et al. [13] evaluated the effect of peppermint aroma on video game performance alongside measures of both subjective and objective arousal. The results indicate that improved game performance was delivered and that this was associated with less subjective demand, effort and anxiety, and greater physiological arousal.

In terms of mechanisms, the impact of peppermint on subjective alertness mediating the effects on cognition has face value but doesn't always hold to close scrutiny. Powell and Moss [24] found no impact on subjective mood in the study where improvements in prospective memory were observed, and no correlation exists between alertness and performance in the data of Moss et al. [15]. Other studies have often implied changes in arousal or alertness to underpin improvements in performance without actually measuring physiological state or subjective mood e.g. Barker et al. [23], or taken a subjective proxy of performance when mood is measured [25]. As a consequence further avenues need to be explored.

Doi et al. [26] provide evidence from a small-scale study indicating the impact of peppermint on haemodynamic measurements using multi-channel near infra-red spectroscopy (NIRS). Compared to no aroma controls they report peppermint as having a large impact that appears quickly and that is maintained for some time following the removal of the aroma. Although no measures of cognition were included in the study the changes reported for the haemodynamic measures coincide with those observed for oral administration of rosemary where associated improvements on cognition were also reported [27]. It may be therefore that improved cognitive performance is a consequence of increased delivery of glucose and oxygen at times of high demand. Similar effects have been noted by supplementation of these metabolic fuels previously [28] [29]. However, the low level extended exposure to peppermint aroma in the current study may seem unlikely to exert the same effects on haemodynamics as the acute effects cited above, although Obrig et al. [30] provide NIRS data that suggest neurovascular coupling is exhibited in healthy adults. Changes may occur and be enhanced by peppermint via a demand-based mechanism. 
A final mechanism that should be considered is one that is pharmacologically based. Rohloff [31] reported on the monoterpene composition of peppermint essential oil and identified 1,8-cineole as the compound in greatest concentration. This may be of particular interest as the compound has been demonstrated to act as an acetylcholinesterase inhibitor [32] [33]. Such a process is the foundation of most drugs designed to counter cognitive decline [34]. Furthermore, serum levels of 1,8-cineole following exposure to rosemary aroma have been shown to correlate with animal activity [35] and cognitive performance in humans [18]. Although not assessed in the current study it might be the case that continuous low level exposure to peppermint aroma delivers increased 1,8-cineole that may have an impact at a neurochemical level. A recent study employing functional magnetic resonance imaging (fMRI) revealed that transdermal application of 1,8 cineole increased activation in a number of brain areas and reduced the number of errors in a working memory task [36]. However, no such effect was found for inhalation. This final observation may appear discouraging in relation to the proposal of a pharmacological mechanism, but the fact that pure 1,8-cineole was chosen as the treatment might be important here. Other components of essential oils-in the case of peppermint menthol and menthone among others-have also been demonstrated to have pharmacological actions [37] [38], and it may be that a synergistic activity is required to deliver maximum benefits; 1,8-cineole alone may be necessary but not sufficient.

\section{Conclusion}

To conclude, the current study adds to the growing body of evidence that the aromas of essential oils can impact on human cognition and mood in the healthy population. Importantly, this is the first time that extended low-level exposure to peppermint aroma has been assessed on a wide range of variables and as such the findings should be treated as preliminary. There is still considerable work to be done to clearly identify mechanisms of action and potential remedial applications through a lens of well-designed scientific rigour. However, small steps and the accumulation of evidence provide the encouragement for future work to be undertaken.

\section{Statement of Funding}

The authors received no funding to support this research.

\section{Conflicts of Interest}

The authors declare that no conflict of interests exist in the production of this paper.

\section{References}

[1] McKay, D.L. and Blumberg, J.B. (2006) A Review of the Bioactivity and Potential Health Benefits of Peppermint Tea (Mentha piperita L.). Phytotherapy Research: An 
International Journal Devoted to Pharmacological and Toxicological Evaluation of Natural Product Derivatives, 20, 619-633. https://doi.org/10.1002/ptr.1936

[2] Sanderson, H. and Ruddle, J. (1992) Aromatherapy and Occupational Therapy. British Journal of Occupational Therapy, 55, 310-314. https://doi.org/10.1177/030802269205500807

[3] Lane, B., Cannella, K., Bowen, C., Copelan, D., Nteff, G., Barnes, K., Lawson, J., et al. (2012) Examination of the Effectiveness of Peppermint Aromatherapy on Nausea in Women Post C-Section. Journal of Holistic Nursing, 30, 90-104. https://doi.org/10.1177/0898010111423419

[4] Toda, M. and Morimoto, K. (2011) Evaluation of Effects of Lavender and Peppermint Aromatherapy Using Sensitive Salivary Endocrinological Stress Markers. Stress and Health, 27, 430-435. https://doi.org/10.1002/smi.1402

[5] Eccles, R., Jawad, M.S. and Morris, S. (1990) The Effects of Oral Administration of (-)-Menthol on Nasal Resistance to Airflow and Nasal Sensation of Airflow in Subjects Suffering from Nasal Congestion Associated with the Common Cold. Journal of Pharmacy and Pharmacology, 42, 652-654.

https://doi.org/10.1111/j.2042-7158.1990.tb06625.x

[6] Norrish, M.I.K. and Dwyer, K.L. (2005) Preliminary Investigation of the Effect of Peppermint Oil on an Objective Measure of Daytime Sleepiness. International Journal of Psychophysiology, 55, 291-298. https://doi.org/10.1016/j.ijpsycho.2004.08.004

[7] Mahachandra, M. and Garnaby, E.D. (2015) The Effectiveness of In-Vehicle Peppermint Fragrance to Maintain Car Driver's Alertness. Procedia Manufacturing, 4, 471-477. https://doi.org/10.1016/j.promfg.2015.11.064

[8] Kwak, S.-H., Seo, S.-H., Kim, H.-S., Chung, S.-C. and Min, B.-C. (2015) 2C4-1 Effects of Sleep Deprivation and the Odor of 100\% Lavender Oiland 100\% Peppermint Oil on Unexpected Situation while Driving. The Japanese Journal of Ergonomics, 51, S500-S505. https://doi.org/10.5100/jje.51.S500

[9] Raudenbush, B., Corley, N. and Eppich, W. (2001) Enhancing Athletic Performance through the Administration of Peppermint Odor. Journal of Sport and Exercise Psychology, 23, 156-160. https://doi.org/10.1123/jsep.23.2.156

[10] Pournemati, P., Azarbayjani, M., Rezaee, M. and Ziaee, V. (2009) The Effect of Inhaling Peppermint Odor and Ethanol in Women Athletes. Bratislavske Lekarske Listy, 110, 782-787.

[11] Basevitch, I., Thompson, B., Braun, R., Razon, S., Arsal, G., Tokac, U., Tenenbaum, G., et al. (2011) Olfactory Effects on Attention Allocation and Perception of Exertion. The Sport Psychologist, 25, 144-158. https://doi.org/10.1123/tsp.25.2.144

[12] Cruz, A.B., TaeHo, K. and SangBum, P. (2010) Effects of Lavender (Lavandula angustifolia Mill.) and Peppermint (Mentha cordifolia Opiz.) Odors on Anxiety and Sport Skill Performance. The Asian International Journal of Life Sciences, 20, 323-329.

[13] McCombs, K., Raudenbush, B., Bova, A. and Sappington, M. (2011) Effects of Peppermint Scent Administration on Cognitive Video Game Performance. North American Journal of Psychology, 13, 383.

[14] Feit, T. and Leopold-Wildburger, U. (2013) Olfactory Effects on Human Behavior within a Simulation Experiment. International Journal of Decision Support System Technology, 5, 49-58. https://doi.org/10.4018/jdsst.2013070104

[15] Moss, M., Hewitt, S., Moss, L. and Wesnes, K. (2008) Modulation of Cognitive Performance and Mood by Aromas of Peppermint and Ylang-Ylang. International Journal of Neuroscience, 118, 59-77. https://doi.org/10.1080/00207450601042094 
[16] Kennedy, D., Okello, E., Chazot, P., Howes, M.-J., Ohiomokhare, S., Jackson, P., Wightman, E., et al. (2018) Volatile Terpenes and Brain Function: Investigation of the Cognitive and Mood Effects of Mentha $\times$ piperita L. Essential Oil with in Vitro Properties Relevant to Central Nervous System Function. Nutrients, 10, 1029. https://doi.org/10.3390/nu10081029

[17] Moss, L., Rouse, M., Wesnes, K.A. and Moss, M. (2010) Differential Effects of the Aromas of Salvia Species on Memory and Mood. Human Psychopharmacology: Clinical and Experimental, 25, 388-396. https://doi.org/10.1002/hup.1129

[18] Moss, M. and Oliver, L. (2012) Plasma 1, 8-cineole Correlates with Cognitive Performance Following Exposure to Rosemary Essential Oil Aroma. Therapeutic Advances in Psychopharmacology, 2, 103-113. https://doi.org/10.1177/2045125312436573

[19] Morris, N. (2002) The Effects of Lavender (Lavendula angustifolium) Baths on Psychological Well-Being: Two Exploratory Randomised Control Trials. Complementary Therapies in Medicine, 10, 223-228. https://doi.org/10.1016/S0965-2299(02)00086-9

[20] Hodge, N.S., McCarthy, M.S. and Pierce, R.M. (2014) A Prospective Randomized Study of the Effectiveness of Aromatherapy for Relief of Postoperative Nausea and Vomiting. Journal of PeriAnesthesia Nursing, 29, 5-11. https://doi.org/10.1016/j.jopan.2012.12.004

[21] Phillips, L.H., Bull, R., Adams, E. and Fraser, L. (2002) Positive Mood and Executive Function: Evidence from Stroop and Fluency Tasks. Emotion, 2, 12. https://doi.org/10.1037/1528-3542.2.1.12

[22] Cohen, J. (1992) A Power Primer. Psychological Bulletin, 112, 155. https://doi.org/10.1037/0033-2909.112.1.155

[23] Barker, S., Grayhem, P., Koon, J., Perkins, J., Whalen, A. and Raudenbush, B. (2003) Improved Performance on Clerical Tasks Associated with Administration of Peppermint Odor. Perceptual and Motor Skills, 97, 1007-1010. https://doi.org/10.2466/pms.2003.97.3.1007

[24] Powell, A. and Moss, M. (2014) Enhancement of Prospective Memory by Peppermint Aroma Is Not Associated with Changes in Subjective Mood State. Proceedings of the British Psychological Society Annual Conference, Birmingham.

[25] Raudenbush, B., Grayhem, R., Sears, T. and Wilson, I. (2009) Effects of Peppermint and Cinnamon Odor Administration on Simulated Driving Alertness, Mood and Workload. North American Journal of Psychology, 11, 245-256.

[26] Doi, S.I., Kamesawa, K., Wada, T., Kobayashi, E., Karaki, M. and Mori, N. (2010) Basic Study on Individual Preference for Scents and the Arousal Level for Brain Activity Using MNIRS. IEEE/ICME International Conference on Complex Medical Engineering, Gold Coast, 13-15 July 2010, 119-124. https://doi.org/10.1109/ICCME.2010.5558859

[27] Moss, M., Smith, E., Milner, M. and McCready, J. (2018) Acute Ingestion of Rosemary Water: Evidence of Cognitive and Cerebrovascular Effects in Healthy Adults. Journal of Psychopharmacology, 32, 1319-1329. https://doi.org/10.1177/0269881118798339

[28] Scholey, A.B., Harper, S. and Kennedy, D.O. (2001) Cognitive Demand and Blood Glucose. Physiology \& Behavior, 73, 585-592. https://doi.org/10.1016/S0031-9384(01)00476-0

[29] Scholey, A.B., Moss, M.C. and Wesnes, K. (1998) Oxygen and Cognitive Performance: The Temporal Relationship between Hyperoxia and Enhanced Memory. 
Psychopharmacology, 140, 123-126. https://doi.org/10.1007/s002130050748

[30] Obrig, H., Israel, H., Kohl-Bareis, M., Uludag, K., Wenzel, R., Müller, B., Villringer, A., et al. (2002) Habituation of the Visually Evoked Potential and Its Vascular Response: Implications for Neurovascular Coupling in the Healthy Adult. Neuroimage, 17, 1-18. https://doi.org/10.1006/nimg.2002.1177

[31] Rohloff, J. (1999) Monoterpene Composition of Essential Oil from Peppermint (Mentha $\times$ piperita L.) with Regard to Leaf Position Using Solid-Phase Microextraction and Gas Chromatography/Mass Spectrometry Analysis. Journal of Agricultural and Food Chemistry, 47, 3782-3786. https://doi.org/10.1021/jf981310s

[32] Orhan, I., Aslan, S., Kartal, M., Şener, B. and Başer, K.H.C. (2008) Inhibitory Effect of Turkish Rosmarinus officinalis L. on Acetylcholinesterase and Butyrylcholinesterase Enzymes. Food Chemistry, 108, 663-668. https://doi.org/10.1016/j.foodchem.2007.11.023

[33] Perry, N.S., Houghton, P.J., Theobald, A., Jenner, P. and Perry, E.K. (2000) In-Vitro Inhibition of Human Erythrocyte Acetylcholinesterase by Salvia lavandulaefolia Essential Oil and Constituent Terpenes. Journal of Pharmacy and Pharmacology, 52, 895-902. https://doi.org/10.1211/0022357001774598

[34] Xie, S.-S., Wang, X., Jiang, N., Yu, W., Wang, K. D., Lan, J.-S., Kong, L.-Y., et al. (2015) Multi-Target Tacrine-Coumarin Hybrids: Cholinesterase and Monoamine Oxidase B Inhibition Properties against Alzheimer's Disease. European Journal of Medicinal Chemistry, 95, 153-165. https://doi.org/10.1016/j.ejmech.2015.03.040

[35] Kovar, K., Gropper, B., Friess, D. and Ammon, H. (1987) Blood Levels of 1, 8-Cineole and Locomotor Activity of Mice after Inhalation and Oral Administration of Rosemary Oil. Planta Medica, 53, 315-318. https://doi.org/10.1055/s-2006-962725

[36] Ambrosch, S., Duliban, C., Heger, H., Moser, E., Laistler, E., Windischberger, C. and Heuberger, E. (2018) Effects of 1, 8-Cineole and (-)-Linalool on Functional Brain Activation in a Working Memory Task. Flavour and Fragrance Journal, 33, 235-244. https://doi.org/10.1002/ffj.3436

[37] Xue, J., Li, H., Deng, X., Ma, Z., Fu, Q. and Ma, S. (2015) L-Menthone Confers Antidepressant-Like Effects in an Unpredictable Chronic Mild Stress Mouse Model via NLRP3 Inflammasome-Mediated Inflammatory Cytokines and Central Neurotransmitters. Pharmacology Biochemistry and Behavior, 134, 42-48. https://doi.org/10.1016/j.pbb.2015.04.014

[38] Oz, M., El Nebrisi, E.G., Yang, K.-H.S., Howarth, F.C. and Al Kury, L.T. (2017) Cellular and Molecular Targets of Menthol Actions. Frontiers in Pharmacology, 8 , 472. https://doi.org/10.3389/fphar.2017.00472 